AperTO - Archivio Istituzionale Open Access dell'Università di Torino

\begin{abstract}
Surgical Implantation of Artificial Urinary Device and Penile Prosthesis Through Trans-scrotal Incision for Postprostatectomy Urinary Incontinence and Erectile Dysfunction: Synchronous or Delayed Procedure?
\end{abstract}

This is the author's manuscript

Original Citation:

Availability:

This version is available http://hdl.handle.net/2318/118566

since

Published version:

DOI:10.1016/j.urology.2012.08.003

Terms of use:

Open Access

Anyone can freely access the full text of works made available as "Open Access". Works made available under a Creative Commons license can be used according to the terms and conditions of said license. Use of all other works requires consent of the right holder (author or publisher) if not exempted from copyright protection by the applicable law. 
This Accepted Author Manuscript (AAM) is copyrighted and published by Elsevier. It is posted here by agreement between Elsevier and the University of Turin. Changes resulting from the publishing process - such as editing, corrections, structural formatting, and other quality control mechanisms - may not be reflected in this version of the text. The definitive version of the text was subsequently published in UROLOGY, 80, issue 5, 2012, 10.1016/j.urology.2012.08.003.

You may download, copy and otherwise use the AAM for non-commercial purposes provided that your license is limited by the following restrictions:

(1) You may use this AAM for non-commercial purposes only under the terms of the CC-BY-NC-ND license.

(2) The integrity of the work and identification of the author, copyright owner, and publisher must be preserved in any copy.

(3) You must attribute this AAM in the following format: Creative Commons BY-NC-ND license (http://creativecommons.org/licenses/by-nc-nd/4.0/deed.en), 10.1016/j.urology.2012.08.003

The publisher's version is available at:

http://linkinghub.elsevier.com/retrieve/pii/S009042951200893X

When citing, please refer to the published version.

Link to this full text:

http://hdl.handle.net/2318/118566 


\section{Surgical Implantation of Artificial Urinary Device and Penile Prosthesis Through Trans-scrotal Incision for Postprostatectomy Urinary Incontinence and Erectile Dysfunction: Synchronous or Delayed Procedure?}

Luigi Rolle, Carlo Ceruti, Omid Sedigh, Massimiliano Timpano, Paolo Destefanis, Beatrice Lillaz, Elisa Galletto, Claudia De Maria, Marco Falcone, Ettore Dalmasso, Dario Fontana

Divisione Universitaria di Urologia II, Dipartimento di Discipline Medico-Chirurgiche, Ospedale "San Giovanni Battista-Molinette," Università degli Studi di Torino, Torino, Italy

\section{Objective}

To compare simultaneous and 2-stage surgical implantation of an artificial urinary sphincter (AUS) and penile prosthesis (PP) for patients with severe urinary incontinence and erectile dysfunction after prostatectomy.

\section{Methods}

The data from 23 patients were reviewed, 15 (group 1) underwent simultaneous implantation and 8 (group 2) underwent 2-stage surgery. The operative time, complications, postoperative pain, incidence of pre- and postoperative urinary incontinence, erectile dysfunction rating (International Consultation on Incontinence questionnaire, Incontinence Quality of Life, International Index of Erectile Function, and Erectile Dysfunction Inventory of Treatment Satisfaction), and patient satisfaction were evaluated.

\section{Results}

The mean operative time was 140 minutes for the simultaneous procedure (group 1) and 105 minutes for AUS placement and 65 minutes for PP placement (group 2). No major postoperative complications were recorded. The average postoperative hospital stay was 2.5 days after the double-implant procedure and 2.4 days after AUS and 1.2 days after PP placement. The visual analog scale score at 6 and 24 hours after surgery was 5 and 3 in group 1, and 4 and 3 after AUS and 3 and 2 after PP (group 2; P > .05). At 1 year, all patients were socially continent, with an average International Consultation on Incontinence score of 2 in group 1 and 2 in group 2 . Of the patients in groups 1 and 2,65\% and 68\% were completely dry, respectively $(P>.05)$. The Incontinence Quality of Life score increased from 37 to 84 in group 1 and from 41 to 82 in group 2. The Patient Global Impression of Improvement revealed that $92 \%$ of group 1 and $95 \%$ of group 2 experienced "great improvement" $(P>.05)$. The International Index of Erectile Function and Erectile Dysfunction Inventory of Treatment Satisfaction score was 70 of 75 and 87 of 100 in group 1 and 72 of 75 and 86 of 100 in group 2, respectively ( $P>.05$ ). Group 1 patients were completely satisfied, and all group 2 patients stated they would have preferred synchronous surgery. 


\section{Conclusion}

AUS and PP synchronous placement is feasible and safe and as effective as the 2-stage procedure, with better acceptance by patients.

Urinary incontinence (UI) and erectile dysfunction (ED) are the most frequent complications affecting the prostatectomy outcome. The treatment of patients affected by both UI and ED is a major urologic problem. The therapy of choice is implantation of an artificial urinary sphincter (AUS) for UI and a penile prosthesis (PP) for ED in selected patients not responding to, or not candidates for, other medical or surgical procedures.

Many investigators have documented the safety, effectiveness, and patient satisfaction for single implantation of an AUS or a PP, with excellent results.1, 2 and 3 In 2003, Wilson et al4 introduced the possibility of performing implantation of an AUS using a single trans-scrotal incision with satisfactory results. Their clinical trial paved the way for simultaneous dual implantation of the AUS and PP through the same scrotal incision.

Few available published studies have demonstrated the feasibility, safety, and cost-effectiveness of dual implantation. Moreover, few have reported encouraging outcomes in functional results and patient satisfaction level.5, 6 and 7 No studies comparing synchronous and delayed implantations of the 2 devices are available.

Our aim was to report our single-center experience comparing simultaneous and 2-stage surgical correction of UI and ED in terms of the surgical feasibility, complications, outcomes, and patient satisfaction.

\section{Material and Methods}

We retrospectively reviewed the clinical and surgical documentation of 23 patients (average age 59 years) who had undergone retropubic radical prostatectomy. From January 2005 to February 2011, these same 23 patients sought treatment of severe UI due to sphincter damage and severe ED, not responsive to phosphodiesterase type 5 inhibitors and not responsive or negatively responsive to intracavernous injection therapy. The preoperative evaluation included cystoscopy and urodynamic studies. Cystoscopy was used to ascertain the patency of the urethra and bladderurethral anastomosis.

UI was assessed preoperatively using the International Consultation on Incontinence (ICI) Questionnaire-Urinary Incontinence Short Form,"8 the Incontinence Quality of Life (I-QoL),9 and "social continence" (defined in our study as the use of no pads or $\leq 1 \mathrm{pad} / \mathrm{d}$ ). The term "social continence" can be defined as the use of 0,1 , or 2 pads daily, depending on the investigators, but most consider $\leq 1 \mathrm{pad} / \mathrm{d}$ to indicate continence.10 and 11 
Sexual dysfunction was determined before surgery using a psychosexual assessment, the International Index of Erectile Function (IIEF), and dynamic Doppler ultrasonography. Sexual dysfunction was assessed 6 months after implantation of the PP using the IIEF and Erectile Dysfunction Inventory of Treatment Satisfaction.

Of the 25 patients, 15 (group 1) underwent simultaneous placement of the AUS and PP (10 patients chose a noninflatable PP and 5 preferred an inflatable PP), and 8 (group 2) underwent deferred surgical correction of UI and ED. Group 2 first underwent implantation of an AUS for severe $\mathrm{UI}$ and, in the second stage, underwent implantation of a nonhydraulic malleable PP. The average period between the first and second procedure was 15 months. AUS/PP implantation surgery was performed 10 months (range 8-13) after radical retropubic prostatectomy in both groups.

In the presence of both severe $\mathrm{UI}$ and ED, our policy was first to treat the main complaint of the patient, typically the UI. Subsequently, many patients, satisfied by their great improvement in terms of continence, exhorted us to solve their sexual dysfunction and, accordingly, underwent PP implantation. During the same period, 3 other patients were treated. These patients were affected by severe ED and mild or very mild UI and considered sexual impotence to be their main problem after prostatectomy. Thus, as the first procedure, these patients requested PP placement and as the second procedure, AUS implantation. One of these patients underwent concomitant PP and sling implantation, reported unsatisfactory improvement of the UI 13 months after surgery. Two other patients experienced an aggravation of UI, 1 year after PP implantation, because of endoscopic revision of the bladder-urethral anastomosis necessary to treat a stenosis. However, the deferred surgical procedure was easily feasible with no significant difficulties with AUS implantation in patients already having a PP or sling. However, we decided not to include this group of patients because it was not statistically relevant owing to the poor number of cases reported.

Considering the number of patients subsequently undergoing the 2 individual procedures, in accordance with previous data reports,4, 5, 6 and 7 we changed our policy by proposing synchronous PP and AUS implantation in patients affected by both ED and UI.

Considering the preoperative severity of incontinence in our patients, AUS implantation was preferred to a sling, as mentioned in the European Association of Urology guidelines.12 The AMS 800 double-cuff artificial urinary sphincter was implanted in all cases. In all our surgical procedures, an AMS CX 700 was used as a hydraulic PP, and a Subrini Virilis II and an AMS Spectra were implanted as noninflatable PP in 15 and 3 cases, respectively.

Both synchronous implantation of the PP and AUS and implantation of the AUS, as an individual procedure, were performed with the same single transversal scrotal incision. In all procedures, the reservoir of the AUS was located intraperitoneally by a second, small pararectal incision. The reservoir of the hydraulic penile implants was always located extraperitoneally, in the retropubic space, by a single penoscrotal incision, in accordance with the technique of Wilson et al.4 All 
patients were extensively informed about the procedures and provided written informed consent for AUS and/or PP implantation.

Postoperative pain was rated using a visual analog score at 6 and 24 hours after each surgical procedure. All patients were evaluated at the 12-month routine follow-up visit after synchronous implantation (group 1) and after the second surgical procedure in group 2. UI was assessed postoperatively using the $\mathrm{ICl}$ questionnaire,8 I-QoL,9 and Patient Global Impression of Improvement.13 Postoperative "social continence" was also rated. ED was evaluated using the IIEF questionnaire. Patient satisfaction with PP implantation was assessed using the Erectile Dysfunction Inventory of Treatment Satisfaction questionnaire.

The data from the 2 groups was statistically analyzed using the SPSS statistical program with the Wilcoxon and chi-square tests.

\section{Results}

The average operative time for the double implantation group (group 1) was 140 minutes. The average operative time was 155 minutes (range 140-180) to implant an inflatable PP and 130 minutes (range 120-150) to implant a malleable PP. In group 2, the average operative time was 105 minutes (range 95-120) for the AUS and 65 minutes (range 60-80) for the PP (all were nonhydraulic implants).

No major intraoperative complications (ie, bleeding, urethral injury, apex or crura perforation) were recorded in either group.

In group 1, the average postoperative hospital stay was 2.5 days. In group 2, the average postoperative stay was 2.4 days after AUS implantation and 1.2 days after PP implantation. We did not find any statistically significant differences in the postoperative hospital stay between the 2 groups.

The average postoperative pain as assessed using the visual analog scale at 6 and 24 hours postoperatively was 5 and 3 in group 1 and 4 and 3 after AUS and 3 and 2 after PP implantation in group 2, respectively. The difference in the subjective assessment of pain using the visual analog scale between the 2 groups was not statistically significant $(P>.05)$.

The average preoperative ICl score was 20 (range 14-21) in group 1 and 18 (range 17-20) in group 2. At the 1-year postoperative follow-up visit, all patients were socially continent, with an average $\mathrm{ICl}$ score of 2 (range 1-5) in group 1 and 2 (range 1-4) in group 2. Moreover, 65\% and 68\% of patients were completely dry in groups 1 and 2, with no significant difference between the 2 groups $(P>.05)$.

The average preoperative I-QoL score was 37 (range 27-51) in group 1 and 41 (range 31-55) in group $2(P=.243)$. At 1 year after surgery, the postoperative I-QoL score was 84 (range 72-89) in 
group 1 and 82 (range 69-88) in group 2, with no meaningful difference between the 2 groups $(P=$ $.533)$.

Our data have suggested that the results in terms of improvement of quality of life are not significantly different whether the UI and ED devices were surgically placed in a simultaneous procedure or a 2-stage procedure.

On the PGI-I, the patients reported experiencing "great improvement" in 92\% in group 1 and 95\% in group 2 at 3 months after surgery. The difference was not statistically significant $(P>.05)$. The remaining $8 \%$ of patients in group 1 and $5 \%$ in group 2 considered themselves to have significant improvement $(P>.05)$.

All patients reported excellent sexual improvement. The IIEF (all domains) and Erectile Dysfunction Inventory of Treatment Satisfaction score was 70 of 75 and 87 of 100 in group 1 and 72 of 75 and 86 of 100 in group 2 , respectively, with no statistically significant differences $(P>.05)$ between the 2 groups.

All group 1 patients were completely satisfied with the single procedure for both their UI and their ED. All patients in group 2 would have chosen a single procedure for correction of both problems if asked again.

One patient in group 1, who had previously undergone salvage external beam radiotherapy for local recurrence of prostate cancer, experienced a sphincter infection after 2 urethrotomies performed for recurrent urethral stricture. The 2 devices were surgically removed 1.5 years after dual implantation (grade IIla). No major postoperative complications were detected in group 2.

\section{Comment}

Some investigators have defined the goals of radical prostatectomy as a "trifecta," including in the definition long-term cancer control and recovery of sexual and urinary function. Although the incidence of ED and $U I$ has generally decreased, they remain the most defying long-term complications of radical prostatectomy.14, 15, 16 and 17 Prosthetic surgery can effectively treat both conditions and represents an additional chance to obtain the trifecta.18

Currently, the placement of a PP is widely considered an effective and safe procedure to regain sexual satisfaction in patients with iatrogenic erectile dysfunction not responding to medical therapy or for patients or unwilling to use medical therapy.1, 2 and 19

The management of $\mathrm{UI}$ is more controversial.2, 20 and 21 The European Association of Urology Guidelines12 consider AUS placement, after a period of conservative management of 6-12 months, as the treatment of choice and the reference standard for intermediate to severe postprostatectomy UI. In contrast, the male sling, considered by some investigators as a satisfactory treatment of postprostatectomy $U \mathrm{UI}, 22$ and 23 has been deemed by the European Association of Urology guidelines as an alternative exclusively for mild to moderate UI. 
The urinary sphincter has been widely used to treat $\mathrm{UI}$ after prostatectomy, with a satisfaction rate of $\leq 90 \%$ and total continence rates of approximately $30 \% .3,4$ and 24

In 1993, Brito et al25 proposed the placement of a second cuff to achieve better outcomes in social continence with excellent results. In a recent report, O'Connor et al26 compared single versus double-cuff AUS implantation, with a significantly greater rate of complete continence and improvement in the Incontinence Impact Questionnaire 7 in men with the double-cuff AUS compared with single-cuff devices. Placing a double-cuff AUS is safe and effective in the treatment of severe postprostatectomy UI,27 with only a slight increase in the operative time. This procedure is standard in our center.

The main unsolved problem regarding the management of both ED and UI after prostatectomy is whether it is better to treat both conditions in a single surgical procedure or in a 2-stage fashion.

Some investigators have suggested treating UI and ED using sling implantation and a PP. In 2005, Rhee28 described a technique for concomitant implantation of an inflatable and semirigid PP with the synthetic male sling, with a high rate of patient satisfaction. Gorbatiy et al29 recently proposed synchronous implantation of an inflatable PP and a bulbourethral sling using a single perineal incision, with satisfactory results.

In 2003, Wilson et al4 reported, for the first time, on AUS placement through a single upper scrotal incision, with good access to the bulbar urethra and the retropubic and subdartos spaces and satisfactory results. Scrotal access paved the way toward synchronous implantation of an AUS and a PP.

In 2008, Henry et al30 evaluated in a retrospective study the differences in terms of continence between the traditional perineal and trans-scrotal approaches. Henry et al30 argued that when an AUS cuff is placed through a perineal approach, a greater completely dry rate and fewer subsequent tandem-cuff additions would be registered compared with the trans-scrotal approach.

Some investigators have tested synchronous prosthetic treatment of male UI and ED with an AUS and a PP, with satisfactory results. Sellers et al5 showed that synchronous double implantation significantly reduced the operative time and was associated with cost savings of approximately at $\$ 7000$ compared with the 2 procedures performed separately. Kendirci et al6 reported on the safety and the effectiveness of dual implantation compared with single implantation in a series of 22 patients, with an average follow-up of 17 months. Their intermediate follow-up results were favorable. According to their report, the inherent advantage of a single anesthetic event and a single trans-scrotal incision should encourage widespread acceptance of this technique. In 2008, Mancini7 reported that the dual implantation produces encouraging outcomes in the patient's satisfaction level and it provides excellent functional results, similar to the placement of a single device alone. 
The synchronous placement of the 2 devices does not present with particular surgical difficulties. As expected, a slightly longer operative time results when positioning an inflatable PP but always within the times considered safe regarding the risk of infection. The postoperative pain was only slightly greater 6 hours after double implantation compared with AUS implantation alone, and the average visual analog scale score was the same after 24 hours.

The incidence of surgical site and prosthesis infections and other complications did not increase. The only case of late (1.5 years) infection of the AUS occurred after an endoscopic procedure in a patient in group 1, and it did not seem likely that is was related to the concomitant placement of the 2 devices.

The functional results at 1 year of follow-up in social/complete continence and as evaluated by validated questionnaires do not show statistically significant differences between the single-stage and 2-stage procedures. Moreover, when asked, our patients preferred the single-stage surgery for the correction of both problems. Even the patients who had undergone a 2-stage procedure, when interviewed, would have preferred the single procedure with double implantation.

\section{Conclusion}

Our data suggest that dual implantation of a PP and an AUS does not result in a greater incidence of intra- or postoperative complications, is well tolerated, has the same long-term functional outcomes of traditional 2-stage surgery, and, furthermore, is much preferred by the patient. PP and AUS synchronous placement is a safe, highly satisfactory, and cost-effective surgery to treat patients affected by both ED and severe UI after radical prostatectomy, allowing the avoidance of multiple procedures and double anesthesia.

\section{References}

F. Montorsi, P. Rigatti, G. Carmignani, et al.

AMS three-piece inflatable implants for erectile dysfunction: a long-term multi-institutional study in 200 consecutive patients

Eur Urol, 37 (2000), pp. 50-55

C.C. Carson, J.J. Mulcahy, F.E. Govier

Efficacy, safety and patient satisfaction outcomes of the AMS 700CX inflatable penile prosthesis: results of a long-term multicenter study: AMS 700CX Study Group

J Urol, 164 (2000), pp. 376-380 
H.H. Lai, T.B. Boone

Implantation of artificial urinary sphincter in patients with post-prostatectomy incontinence, and preoperative overactive bladder and mixed symptoms

J Urol, 185 (2011), pp. 2254-2259

4

S.K. Wilson, J.R. Delk II, G.D. Henry, et al.

A new approach for implantation of artificial urinary sphincter: upper transverse scrotal incision

J Urol, 169 (2003), pp. 261-264

5

C.L. Sellers, A.F. Morey, L.A. Jones

Cost and time benefits of dual implantation of inflatable penile and artificial urinary sphincter prosthetics by single incision

Urology, 65 (2005), pp. 852-853

6

M. Kendirci, S. Gupta, K. Shaw, et al.

Synchronous prosthetic implantation through a transscrotal incision: an outcome analysis

J Urol, 175 (2006), pp. 2218-2222

7

J.G. Mancini, W.S. Kizer, L.A. Jones, et al.

Patient satisfaction after dual implantation of inflatable penile and artificial urinary sphincter prostheses

Urology, 71 (2008), pp. 893-896

8

K. Avery, J. Donovan, T.J. Peters, et al.

ICIQ: a brief and robust measure for evaluating the symptoms and impact of urinary incontinence Neurourol Urodyn, 23 (2004), pp. 322-330 
D.L. Patrick, M.L. Martin, D.M. Bushnell, et al.

Quality of life of women with urinary incontinence: further development of the Incontinence Quality of Life instrument (I-QOL)

Urology, 53 (1999), pp. 71-76

10

E. Gnessin, P.M. Livne, J. Baniel, et al.

Continence and quality of life assessment after artificial urinary sphincter implantation

Isr Med Assoc J, 6 (2004), pp. 592-594

11

P.H. Meulen, V. Zambon, A.G. Kessels, et al.

Quality of life, functional outcome and durability of the AMS 800 artificial urinary sphincter in patients with intrinsic sphincter deficiency

Urol Int, 71 (2003), pp. 55-60

12

J.W. Thüroff, P. Abrams, K.E. Andersson, et al.

EAU guidelines on urinary incontinence

Eur Urol, 59 (2011), pp. 387-400

13

S. Srikrishna, D. Robinson, L. Cardozo

Validation of the patient global impression of improvement (PGI-I) for urogenital prolapse

Int Urogynecol J, 21 (2010), pp. 523-528

14

A. Gallina, M. Ferrari, N. Suardi, et al.

Erectile function outcome after bilateral nerve sparing radical prostatectomy: which patients may be left untreated?

J Sex Med, 9 (2012), pp. 903-908 
A.D. Martin, L.Y. Nakamura, R.N. Nunez, et al.

Incontinence after radical prostatectomy: a patient centered analysis and implications for preoperative counseling

J Urol, 186 (2011), pp. 204-208

16

V. Ficarra, G. Novara, W. Artibani, et al.

Retropubic, laparoscopic, and robot-assisted radical prostatectomy: a systematic review and cumulative analysis of comparative studies

Eur Urol, 55 (2009), pp. 1037-1063

17

F. Montorsi, A. Briganti, A. Salonia, et al.

Current and future strategies for preventing and managing erectile dysfunction following radical prostatectomy

Eur Urol, 45 (2004), pp. 123-133

18

F.J. Bianco Jr, P.T. Scardino, J.A. Eastham

Radical prostatectomy: long-term cancer control and recovery of sexual and urinary function ("trifecta")

Urology, 66 (5 suppl) (2005), pp. 83-94

19

K. Hatzimouratidis, E. Amar, I. Eardley, et al.

Guidelines on male sexual dysfunction: erectile dysfunction and premature ejaculation

Eur Urol, 57 (2010), pp. 804-814

20

R.M. Bauer, C. Gozzi, W. Hübner, et al.

Contemporary management of postprostatectomy incontinence

Eur Urol, 59 (2011), pp. 985-996 
A. Kumar, E.R. Litt, K.N. Ballert, et al.

Artificial urinary sphincter versus male sling for post-prostatectomy incontinence-what do patients choose?

J Urol, 181 (2009), pp. 1231-1235

22

R.M. Bauer, M.E. Mayer, C. Gratzke, et al.

Prospective evaluation of the functional sling suspension for male postprostatectomy stress urinary incontinence: results after 1 year

Eur Urol, 56 (2009), pp. 928-933

23

J.S. Han, B.M. Brucker, A. Demirtas, et al.

Treatment of post-prostatectomy incontinence with male slings in patients with impaired detrusor contractility on urodynamics and/or who perform Valsalva voiding

J Urol, 186 (2011), pp. 1370-1375

24

G.E. Leach, B. Trockman, A. Wong, et al.

Post-prostatectomy incontinence: urodynamic findings and treatment outcomes

J Urol, 155 (1996), pp. 1256-1259

25

C.G. Brito, J.J. Mulcahy, M.E. Mitchell, et al.

Use of a double cuff AMS800 urinary sphincter for severe stress incontinence

J Urol, 149 (1993), pp. 283-285

26

R.C. O'Connor, G.S. Gerber, D. Avila, et al.

Comparison of outcomes after single or double-cuff artificial urinary sphincter insertion Urology, 62 (2003), pp. 723-726 
J.J. Kowalczyk, D.L. Spicer, J.J. Mulcahy

Long-term experience with the double-cuff AMS 800 artificial urinary sphincter

Urology, 47 (1996), pp. 895-897

28

E.Y. Rhee

Technique for concomitant implantation of the penile prosthesis with the male sling

J Urol, 173 (2005), pp. 925-927

29

V. Gorbatiy, O.L. Westney, C. Romero, et al.

Outcomes of simultaneous placement of an inflatable penile prosthesis and a male urethral sling through a single perineal incision

J Sex Med, 7 (2 Pt. 1) (2010), pp. 832-838

30

G.D. Henry, S.M. Graham, M.A. Cleves, et al.

Perineal approach for artificial urinary sphincter implantation appears to control male stress incontinence better than the transscrotal approach

J Urol, 179 (2008), pp. 1475-1479 